\title{
Magnetic Fields of Chemically Peculiar and Related Stars. VII. Main Results of 2020 and Near-Future Prospects
}

\author{
I. I. Romanyuk* \\ Special Astrophysical Observatory, Russian Academy of Sciences, Nizhnii Arkhyz, 369167 Russia \\ *e-mail: roman@sao.ru \\ Received July 22, 2021; revised August 9, 2021; accepted August 9, 2021
}

\begin{abstract}
We present a review of the papers written in the field of stellar magnetism studies in 2020. More than 70 original papers related to different areas of the specified scientific topics have been analyzed. Instruments, methods of analysis, and software are considered, as well as: chemically peculiar stars and their identification; magnetic fields, chemical abundance, and photometry of chemically peculiar stars; magnetic white dwarfs, active cool stars and other objects. In the field of stellar magnetism research, 6-7 scientific groups continue to work actively using the world's largest telescopes, which fact indicates the relevance and importance of the problem under study.
\end{abstract}

Keywords: publications, bibliography

DOI: $10.1134 / \mathrm{S} 1990341321040118$

\section{INTRODUCTION}

This paper continues the series of annual reviews Romanyuk $(2015,2020)$, in which the research results of the magnetic field of chemically peculiar and related stars are published. This paper is dedicated to describing the results presented in the leading journals mainly in 2020 .

Unfortunately, in 2020, the whole world was hit with the coronavirus pandemic, so almost all international astronomical conferences were canceled or postponed to a later date. Of the conferences conducted, the all-Russia conference "Ground Based Astronomy in Russia. 21st century" should be noted held at SAO RAS on September 21-251. Its results are published in the self-titled proceedings edited by I. Romanyuk, I. Yakunin, A. Valeev, and D. Kudryavtsev (Romanyuk et al., 2020e). Here we give consideration to some of these stellar magnetism publications. In addition, this review analyzes the results presented at two conferences held earlier, the proceedings of which were published in 2020:

- "Stars and their Variability, Observed from Space", held in Vienna in August 2019, edited by Neiner, Weiss, Baade et al.;

- "Stellar magnetism: A Workshop in Honour of the Career and Contributions of John Landstreet", held on 8-11 July 2019 in London, Canada, edited by Wade, Alecian, Bohlender, and Sigut.

\footnotetext{
${ }^{1}$ See more on the conference web page: http://jet.sao.ru/conferences $/ 2020 /$.
}

We preserve the order of the sections traditional for these reviews: tools, methods of analysis and software; chemically peculiar stars and their identification; magnetic field, chemical abundance and photometry; magnetic white dwarfs, active cool stars and other objects.

\section{METHODOLOGICAL ISSUES}

\subsection{New Telescopes and Mounted Instruments}

In 2020, relatively few papers have been published on the development and implementation of new telescopes and spectrographs.

The collaboration of more than 100 coauthors presented the HIRES: the high-resolution spectrograph at optical and near-infrared (NIR) wavelengths for ESO's Extremely Large Telescope (ELT) (Marconi et al., 2021). The final device will provide the wavelength range of $0.4-1.8 \mu \mathrm{m}$ with the spectral resolution $R=100000$. Several observing modes will be available including a single-conjugate adaptive optics (SCAO) module and a small diffraction-limited integral field unit in the near-IR. The HIRES will cover a wide range of astrophysical and theoretical physics problems. Some of the most important are the search for signs of life in exoplanet atmospheres and testing the stability of Nature's fundamental couplings. The HIRES team unites more than 30 institutes from 14 countries with more than 200 scientists and engineers.

The spectropolarimetric mode for the ELT-HIRES is under development (Di Varano et al., 2020). It will consist of two assemblies. The main module, consist- 
ing of a double Wollaston calcite prism and the retarder plates splitting the optical beam into the four Stokes vector components, will be installed in the intermediate focus. The secondary one, providing the atmospheric dispersion correction and field stabilization, will be located in one of the Nasmyth platforms. The expected sensitivity is better than hundredths of a percent of the Stokes vector. Various instrumental parameters are investigated including crystal anisotropic properties of the polarizing components, polarimetric aberrations induced by the active compensation of the primary mirror, etc.

Fiber-fed high-resolution spectroscopy is also developing at the 6-m telescope of SAO RAS (Valyavin et al., 2020a, b). The first test observations were carried out with the new spectrograph. As a result, the radial velocity measurement accuracies from several $\mathrm{m} \mathrm{s}^{-1}$ to several tens of $\mathrm{m} \mathrm{s}^{-1}$ were obtained with $R \sim 70000$. With seeing better than $1 "$, the peak efficiency of the optical system reaches $8 \%$ at $6200 \AA$. The coming upgrades include the implementation of spectropolarimetric mode for detecting stellar magnetic fields with up to $1 \mathrm{G}$ accuracy.

Burlakova et al. (2020) present the results of observations of radial velocity variability with the above mentioned spectrograph carried out in high-resolution mode $(R=45000-65000)$ with a simultaneous registration of the calibration spectrum of a Th-Ar hollow cathode lamp. The achieved characteristic measurement accuracies ranged from a few to several tens of $\mathrm{m} \mathrm{s}^{-1}$. In the future, the thorium-argon lamp will be supplemented with a Fabri-Perot etalon to reach the radial velocity measurement accuracy in stars up to $1 \mathrm{~m} \mathrm{~s}^{-1}$.

For the STELLA robotic observatory, the second generation spectroscopic instruments were designed (Weber et al., 2020). The current spectrograph SES, which detects 390 to $870 \mathrm{~nm}$ in one shot with the spectral resolution $R=55000$, will be replaced by a suite of specialized spectrographs in three spectral bands: UV-3800-4700 Å; visual-4700-6900 Å; near-IR6900-10500 $\AA$. The primary focus will be renewed introducing the $f / 21200 \mathrm{~mm}$ spherical primary mirror and a 4-lens collimator with the 2 aperture, atmospheric dispersion corrector, and two dichroic beam splitters feeding 3 separate fiber feeds for three bands.

Donati et al. (2020) introduced SPIRou, the newgeneration near-IR spectropolarimeter/precision velocimeter for the 3.6-m Canada-France-Hawaii Telescope. The developers declare the main science goals as a searching for exoplanetary systems around M dwarfs and the study of magnetized star/planet formation. The SPIRou Legacy Survey (SLS) Large Programme was allocated 300 CFHT nights until at least mid-2022. In addition to exoplanet studies, various science topics include magnetic fields of young stars, the atmospheres of M dwarfs, the Earth's atmosphere, etc. Hobson et al. (2021) describe the wavelength calibration of SPIRou in the hear-IR region (9800$23500 \AA$ A). The UNe hollow-cathode and FabryPerot etalon are used for this. The combination of both methods allowed one to reach an internal error of the radial velocity measurement up to $15 \mathrm{~cm} \mathrm{~s}^{-1}$.

\subsection{Data Reduction and Analysis Methods}

Piskunov et al. (2021) developed and introduced a new optimal extraction technique for echelle spectra. In the world of constantly increasing price of science instruments and observing time on telescopes, it is of especial importance to revisit the data reduction algorithms to extract every bit of scientific information. Regular attempts to improve the wavelength coverage and versatility of high-resolution echelle spectrographs require modernization of the reduction software. The authors propose new algorithms to minimize the resolution losses and increase of the $S / N$ ratio. A comparison of the described method with other techniques clearly illustrate the superior performance of the new algorithms.

Bowman and Holdsworth (2019) created a software package manly for the analysis of oscillations and confirming a rapid variability of new variable stars discovered with modern space telescopes. A significant advantage of the method of the adaptive elliptical aperture photometry are demonstrated in searching for rapid oscillations in roAp candidates observed at the South African Astronomical Observatory (HD 158596, HD 166542, and HD 181810). The authors demonstrate the significant improvement in the analysis of short-period variability of such objects.

Holdsworth and Brunsden (2020) tested the capabilities of the High Resolution Spectrograph (HRS) at the Southern African Large Telescope (SALT) for the study of pulsations in roAp stars. As an example, the results of observations of a bright roAp star $\alpha$ Cir are demonstrated. Using the cross-correlation method, high-precision measurements of the radial velocity were carried out using the lines of different rare-earth elements and in the core of the $H_{\alpha}$. Good agreement with previous observations of this star was obtained, and the second pulsation mode was found. The SALT telescope is adapted to this kind of task.

\section{SPECTROSCOPY AND PHOTOMETRY RESULTS FOR CP STARS}

\subsection{Spectroscopy of CP Stars}

Alexeeva et al. (2020) studied the NLTE-effects of the $\mathrm{Ne}$ I and Ne II lines in the atmospheres of B stars in the solar neighborhood. The large NLTE strengthening of the Ne I lines are found that lead to strong overpopulation of these levels relative to their LTE populations, while deviations from LTE for most Ne II 
lines are small. They analyzed 20 lines of $\mathrm{Ne} \mathrm{I}$ and 13 lines of Ne II for 24 B-type stars in the temperature range from 10000 to $33000 \mathrm{~K}$. The average neon abundance in $24 \mathrm{~B}$-type stars in the solar neighborhood is $\log \epsilon=8.02 \pm 0.05$.

Nielsen et al. (2020) presents the results of the study of the B-type chemically peculiar star HR 465. HR 465 shows unusual abundance patterns that vary with a period of $P=21.5 \mathrm{yrs}$. The spectra for the analysis were obtained at $\phi=0.45, \phi=0.68$, and $\phi=0.85$ of the rotation period, where $\phi=0.0$ corresponds to the $U$-band maximum. The visual spectra obtained with the SOFIN spectrograph at Nordic Optical Telescope and high-resolution ultraviolet Hubble Space Telescope spectra were fitted by synthetic spectra computed using an LTE atmosphere model. Abundances for more than 70 atoms/ions replicate the previously reported, where the elements with high-mass numbers show significant abundance enhancements compared to the solar values, while some of the lighter elements show abundance deficiencies.

Catanzaro et al. (2020) present a detailed spectroscopic study of the SB1 system HD 161660. Atmospheric parameters such as temperature and surface gravity, microturbulence and rotational velocity, and chemical composition were driven using the data from the Catania Astrophysical Observatory Spectropolarimeter and archival data from HARPS/ESO. The analysis showed underabundances of helium, carbon, magnesium, sulphur, and chromium, overabundances of neon, phosphorus, argon, manganese, xenon, and mercury, and a solar composition of the others. Presumably, the star HD 161660 is a HgMn star. Iron and phosphorus abundances show a dependence with the optical depth evidencing a vertical stratification.

Schöller et al. (2020) report the detection of infrared companion of the well-known chemically peculiar star HD 94660. Earlier, some authors believed that the satellite could have a mass over $M=2 M_{\odot}$ and be a compact object. The results of observations in the $H$ band with the VLT interferometer, TESS photometric series, as well as X-ray observations with Chandra are analyzed, and it is assumed that the HD 94660 satellite is not a compact and single object. Most likely, this is a pair of late F stars, whose orbital rotation leads to the observed periodic photometric variability.

Romanovskaya and Shulyak (2019) investigated the chemical abundance and stratification of elements in the atmosphere of the chemically peculiar star HD 110066. They found that the spectrum contains the most numerous lines of iron and chromium, and for them there is a stratification effect. The chemical abundance is typical for this type of stars. The distribution of iron and chromium is generally consistent with the predictions of the diffusion theory. The paper by Romanovskaya et al. (2020) presents the results of determining the atmospheric parameters of HD 110066 and HD 153882. The values of effective temperature, surface gravity, radii and luminosity are derived. The radial components of the magnetic field were determined from the line splitting: $B_{r}=4015 \pm 180 \mathrm{G}$ for HD $110066, B_{r}=3800 \pm 200 \mathrm{G}$ for HD 153882 .

To test the models used to determine the radii and effective temperatures of stars, Perraut et al. (2020) suggested measuring their angular diameters using long-baseline interferometry and then comparing the results with the spectroscopic data. The observations were carried out on the VEGA spectrograph of the CHARA interferometer. As a result of this work, the number of Ap stars, for which the angular diameters were determined from the interferometric data, increased to 14. For 9 Ap stars, with the effective temperatures from 7200 to $9100 \mathrm{~K}$ and luminosities in the range of $7 L_{\odot}-86 L_{\odot}$, the differences obtained by the two methods do not exceed $2 \sigma$. Good agreement between the results is key for the future derivation of accurate radii and other fundamental parameters of fainter peculiar stars for which both the sensitivity and the angular resolution of the current interferometers are not sufficient.

Kholtygin et al. (2020) studied the fast spectral variability of the $H_{\beta}$ line profiles in the spectrum of $\alpha^{2}$ $\mathrm{CVn}$. The observed data were obtained with the 6-m telescope of the SAO RAS using the Main Stellar Spectrograph. Quasiperiodic variability was found at the periods from 4 to 140 minutes. The value of the longitudinal magnetic field determined by the authors coincides with the estimate found from the phase curve.

\subsection{Photometry of CP Stars}

Paunzen et al. (2020) proposes a new project aimed to obtain a homogeneous sample of non-variable stars for various astrophysical purposes. Although there are more than one million photometric light curves to date, there is no sample of permanent stars.

Skarka et al. (2020) report the discovery of a chemically peculiar Ap star with silicon, titanium, and rare earth elements excess concentrated in spots. In addition, the star shows variability of the $\delta$ Sct type which was not previously observed in Ap stars. The star is in a binary system, its companion is a red dwarf which is also very rare.

According to ASAS-3, KELT, and MASCARA data, Bernhard et al. (2020) have refined the rotation periods of magnetic CP stars. A large observation program was carried out. The final sample included 294 objects, of which more than $40 \%$ are photometrically variable objects. In addition, the authors of this work identified 24 more CP candidates. The light curves of these objects are in agreement with the rotational modulation, but there is no spectral data. The 
distribution of rotation periods is consistent with the previously known literature data. The ages of most objects are in the range from 100 Myr to 1 Gyr, very young stars are absent. Four eclipsing binaries are found: HD 244931, HD 247441, HD 248784, and HD 252519. This result is of great interest, since CP stars are very rare in close binaries, especially in eclipsing ones.

Hümmerich et al. (2020) present the results of the search for magnetic chemically peculiar stars based on the data from the LAMOST spectroscopic survey. Candidates were selected based on the presence of depression in the spectra at a wavelength of $5200 \AA$. The GAIA parallaxes were used to analyze the spatial distribution of objects and their properties on the color-magnitude diagram. The final sample contains 1002 CP stars, most of which are new discoveries, since only 59 objects are in the catalogs of peculiar stars. The ages of all the objects are in the range from $100 \mathrm{Myr}$ to $1 \mathrm{Gyr}$, the masses are from $2 M_{\odot}$ to $3 M_{\odot}$. As a result of this paper, the sample of the known $\mathrm{CP}$ stars in our Galaxy has been significantly increased.

Mikulášek et al. (2020b) study the star HD 174356, for which accurate photometric curves were obtained on the Kepler satellite. It turned out that the amplitude and shape of the light curve is not consistent with that expected for CP stars. The spectral analysis shows that HD 174356 is a typical chemically peculiar star with strong silicon lines. The upper limit for the the magnetic field strength is $110 \mathrm{G}$. The light curve can be described by the sum of two independent periodic signals and has not changed over 17 years of observations. It was concluded that HD 174356 is a single star, and its light variability can be explained by the rotational modulation of the inhomogeneous surface and pulsations in the g-mode.

\section{MAGNETIC FIELD OF STARS}

\subsection{Magnetic Field of CP Stars}

To verify the recent report that the magnetic $\mathrm{O}$ star HD 54879 has radial velocity variations and abrupt magnetic field strength changes, Wade et al. (2020) examined new data from high-resolution NARVAL and ESPaDOnS spectrographs, as well as archival FORS2 spectra, and came to the conclusion that the previously detected deviations found for the star were erroneous.

Niemczura et al. (2020) shows that HD 66051 has an extremely peculiar spectrum of the main component. HD 66051 is a very rarely observed case of an eclipsing spectroscopic binary (SB2), in which both components are chemically peculiar stars. The main component is an anomalously strong peculiar B star, the secondary component is an Am star. The TESS data was used to clarify the physical characteristics of the stars and the orbital parameters of the system.
Hubrig et al. (2020b) demonstrated that the O star HD 54879 rotates very slowly. According to FORS2 observations in 2014, its longitudinal magnetic field strength is $B_{e}=-600 \mathrm{G}$. Spectropolarimetric monitoring showed that from October 2017 until February 2018 the longitudinal field strength decreased from -300 to -90 G. New spectropolarimetric observations carried out over two months in 2018-2019 also showed slow field variations. The authors believe that the reason for this is the slow rotation of the star.

A rather strong magnetic field was found in several young stars when searching for the magnetic field in massive stars in the star-forming regions (Hubrig et al., 2020d). According to one of the ideas, it has a fossil nature: the magnetic field of the interstellar medium (ISM) of the Galaxy is enhanced during the collapse of a magnetized gas cloud. Spectropolarimetric observations can help us gain insight into the mechanisms that lead to strong magnetic fields during massive stars' formation stage.

Hubrig et al. (2020a) found a weak magnetic field in two HgMn stars, HD 221507 and HD 65949, in the same epoch; another star, HD 101189, is suspected of having a field. As a rule, such stars do not have magnetic fields or it is probably very weak.

The same team (Järvinen et al., 2021) discusses the geometry of the magnetic field of highly magnetized Of?p star NGC 1624-2. A longitudinal field of greater than $20 \mathrm{kG}$ was found which is the strongest among all $\mathrm{O}$ stars. The geometry of the magnetic field is difficult to reproduce, since only one pole can be observed per rotation cycle. Apparently, in the first approximation, the field can be considered dipole, since the variations of the longitudinal field are sinusoidal. The paper considers different models. Based on the results of observations of the same object with the Hubble Space Telescope, another international team of researchers (David-Uraz et al., 2021) found that the star NGC 1624-2 has a magnetosphere of a complex structure. Spectropolarimetric observations show that the structure of the surface field is also complex.

Petit et al. (2019) reviews the results of the MiMeS program of searching for the magnetic field of O stars. It was found that the distribution of the magnetic field in these objects is bimodal: there are stars with a very strong field, there are stars with a weak or absent field. The structure of the magnetic field is predominantly of a dipole structure. The MiMeS review cannot confirm or deny a magnetic flux decay in O stars.

The joint MiMeS and BINAMICS collaboration presented a new paper (Shultz et al., 2019b). It investigates Main Sequence B stars: their magnetic field, rotation, and parameters of the magnetosphere. The stellar wind is near early B stars. The magnetic confinement of stellar winds leads to the formation of magnetospheres which can be sculpted into centrifugal magnetospheres. 
Using new methods for estimating the rotation period, new magnetic measurements and determining atmospheric parameters, they found physical parameters and dipole field models for 56 early B stars. Comparison with magnetic $\mathrm{O}$ and $\mathrm{A}$ stars shows that the limits of the magnetic field for them are constant, but the magnetic flux increases with mass. The magnetic dipole strength and the magnetic flux decrease with age. The authors found evidence that a multi-polar magnetic field should decay faster than a dipole one. Rotational periods increase with stellar age. All stars with the $H_{\alpha}$ emission are

(1) rapid rotators;

(2) strongly magnetized;

(3) young.

This fact is consistent with the observation that magnetic fields and rotation both decrease over time.

Bagnulo et al. (2020) made an attempt to detect a magnetic field of the brightest massive stars in the Large and Small Magellanic Clouds. A FORS2 spectropolarimeter was used to observe a sample of 41 objects: B, O, Of/WN, WMh, and classical WR stars. Four objects from the list turned out to be Of?p stars. Formally, none of the studied objects has a field detected, however, one of the stars has a longitudinal field of the order of $2-4 \mathrm{kG}$.

Shultz et al. (2019a) describe the results of the study of the magnetic Bp star HD 62658, an eclipsing binary with two identical non-magnetic components. The light curves of the system were obtained from the KELT and TESS satellites. They show clear eclipses with a period of 4.75 days. High-resolution circular spectropolarimetry obtained with ESPaDOnS shows clear signatures of the Zeeman effect for the main component and no indication of a magnetic field for the secondary. The existence of such systems, based on the fossil nature of the magnetic field, cannot be explained by environmental conditions alone.

Keszthelyi et al. (2020) studied the effect of the evolution of the fossil magnetic field for massive stars. The evolution of the angular momentum and rotation of massive stars is highly dependent on the magnitude of the initial field and magnetic braking. Two scenarios have been developed. According to the conclusions of the paper, the magnetic braking on the whole has a small value, making it difficult to infer the initial rotation rates of the evolved, slowly rotating stars.

Blazère et al. (2020) modeled the geometry of the magnetic field and surface differential rotation of the bright Am star Alhena A ( $\gamma$ Gem A). Among Am stars, this object has the strongest surface magnetic field. The paper presents the results of magnetic monitoring carried out for 25 nights on the NARVAL spectropolarimeter. The authors confirm that Alhena A is a magnetic star, the oblique rotator model is suitable for explaining the variability of the longitudinal component. The field at the pole is about $30 \mathrm{G}$. The rotation period of the star is 8.975 days. The ZDI inversion suggests that the surface magnetic field is sheared by differential rotation.

Glagolevskij (2020) reviewed the studies of stars with anomalous helium lines. The results are derived from more than 160 measurements of magnetic field, rotation speed, and other parameters of A stars. The author states that there are still insufficient observed data for reliable conclusions.

Romanyuk et al. (2020c) presented the results of magnetic field measurements for $8 \mathrm{CP}$ stars in the Kepler field using the spectropolarimeter of the 6-m SAO RAS telescope. Five stars (KIC 4180396, KIC 5264818, KIC 5473826, KIC 6065699, and KIC 8324268) were first found as magnetic. It is highly probable that KIC 6864569 is also a magnetic star, but additional observations are required for confirmation. The status of KIC 816798 and KIC 10324412 remains undefined, while KIC 6278403 has no magnetic field detected.

Romanyuk et al. (2020d) presented the results of measurements of the magnetic field of stars with the 6m SAO RAS telescope. Observations were carried out in 2012 using the Main Stellar Spectrograph (MSS) equipped with a Zeeman analyzer. The longitudinal magnetic fields and radial velocities were measured for 163 objects, mainly chemically peculiar stars. Six new magnetic stars have been discovered (HD 84882, HD 109030, HD 170054, HD 189775, HD 341037, and BD +612436). New data were obtained for 120 magnetic stars and 43 non-magnetic ones. The radial velocity of all the objects was measured, 46 stars were found to be binaries. The paper provides comments for each of the 163 stars.

After analyzing 459 measurements of the longitudinal magnetic field of the Ap star $\gamma$ Equ, Savanov et al. (2020) found that the period of its rotation is approximately 101 years. Another variability period of the longitudinal field is possible, about 16.7 years.

Semenko et al. (2020) presented a modern survey of the chemically peculiar star population in the Ori OB1 association. The authors have collected over 500 spectra for more than 60 stars obtained with the 6 - $\mathrm{m}$ telescope. More than 20 new magnetic stars and about 20 binaries have been found.

Shultz et al. (2020a) studied the evolution of the rotation periods of magnetic stars in the Orion association. The angular momentum loss is expected, but so far only a few stars have accurate rotation periods. It is necessary to increase the sample of objects.

Mikulášek et al. (2020a) discuss new results obtained for the unique Landstreet's star HD 37776. Doppler-Zeeman imaging revealed that the stellar surface magnetic field is very strong (more than $30 \mathrm{kG}$ ) and has a complex non-dipole structure. The TESS data of unprecedented high accuracy revealed many features in the light curve that are very difficult 
to reproduce using a standard model with chemical and photometric spots and solid rotation.

Romanyuk et al. (2020b) presented the main results of stellar magnetic field studies carried out over the past 15 years with the 6-m SAO RAS telescope. More than 200 chemically peculiar stars have been observed, the magnetic field has been found in about 80 objects. Special attention was paid to the study of magnetic stars in the Orion OB1 association. More than 500 Zeeman spectra were obtained for 55 stars, 10 new stars were discovered as magnetic. The authors found very long rotation periods of 3.7 years for HD 18078, 29 years for HD 50169, and 17 years for HD 965. The conclusion is made that the SAO RAS magnetic measurement system is stable for 40 years and corresponds to the international one.

To search for the radial gradient of the magnetic field of chemically peculiar stars, measurements were carried out with a Zeeman analyzer in the region of 3500-4200 Å (Romanyuk et al., 2020a). The first results were obtained for the stars $\alpha^{2} \mathrm{CVn}$ and $53 \mathrm{Cam}$.

Romanyuk et al. (2021) presented magnetic field measurements for 15 chemically peculiar stars of subgroup $1 \mathrm{~b}$ in the Orion OB1 association. The occurrence of stars with strong magnetic fields among these $15 \mathrm{CP}$ stars is almost twice as large as in subgroup 1a. The average root-mean-square magnetic field $\left\langle B_{e}\right\rangle$ for the stars in subgroup $1 \mathrm{~b}$ is 2.3 times higher than that for the stars in subgroup 1a. The conclusions obtained fall within the concept of the fossil origin of large-scale magnetic fields in B and A stars, but the rate of field weakening with age appears anomalously high.

The TESS photometry of the magnetic chemically peculiar star HD 65987, a member of the open cluster NGC 2516, shows clear photometric variability with a period of 1.45 days (Landstreet et al., 2020). However, new magnetic and spectroscopic measurements of this star reveal a magnetic field varying with a stellar rotation period of about 7.7 days. The origin of the 1.45 -day variations is suggested to be stellar pulsations.

Mathys et al. (2020) studied the magnetic field of a rapidly oscillating strongly magnetized Ap star HD 166473. On the basis of 56 measurements of the magnetic field modulus, carried out in the period of 1992-2019, the rotation period of the star was found with high accuracy and equaled 3836 days. Thus, it is one of four Bp stars with rotation periods exceeding 10 years. The field of the star is slightly different from a dipole one, but on the whole there is no fundamental difference in the magnetic properties of HD 166473 and more rapidly rotating Ap stars. However, when considering a group of $8 \mathrm{Ap}$ stars with periods of more than 1000 days, for which a complete magnetic cycle has been prescribed, it can be concluded that the angle between the dipole axis and the axis of rotation tends to be systematically large.
Shultz et al. (2020b) are studying the $H_{\alpha}$ emission as a tool to diagnose plasma transition into the centrifugal magnetospheres in rapidly rotating early-type stars with strong magnetic fields. A detailed study of the behavior of the $H_{\alpha}$ emission line makes it possible to check the mechanisms of such a process. Various scenarios are considered. It is shown that the radius of the emission maximum correlates with the Keplerian co-rotation radius, as is expected from the models.

According to the TESS data, coherent pulsation mode was found for the Of?p star NGC 1624-2 (Kurtz et al., 2020). This star is of particular interest, as it has the strongest magnetic field of all $\mathrm{O}$ stars. Three lowfrequency peaks were found, probably associated with the g-mode of the pulsations. To date, this is the only $\mathrm{O}$ star that has been found to exhibit pulsation variability.

\subsection{Magnetic White Dwarfs}

Landstreet and Bagnulo (2020) report the discovery of a unique non-interacting binary system, like Sirius, containing the white dwarf WDS J03038 + 0608B with a very strong (hundreds of MGs) magnetic field and a main sequence star forming a wide pair. The amplitude of the circular polarization in the continuum of the white dwarf is $5 \%$ with no signs of variability on a time scale of 1 day. In the blue region of the spectrum, linear polarization was not detected, and in the red it reaches $2 \%$. Binary non-interacting (widely separated) systems, consisting of a magnetic white dwarf and a Main Sequence star, are considered very rare. Four more such systems are known, which makes it possible to estimate the fraction of magnetic white dwarfs that can be included in wide pairs as few percents. Due to the wide separation, which excludes the evolution stage with filling of the Roche lobe, they cannot be considered as precursors of magnetic cataclysmic variables (such as AM Her and DQ Her).

Bagnulo and Landstreet (2020) discovered 6 new magnetic white dwarfs with a strong magnetic field ranging from several $\mathrm{MG}$ to $200 \mathrm{MG}$ inside a local region of $20 \mathrm{pc}$ in size. Two of these stars show the $\mathrm{H}$ lines that are split and polarized by the magnetic field. The lines in the spectrum of another star, presumably a DA white dwarf, are very faint. The other three stars have featureless spectra, but the continuum is polarized. These discoveries support the idea that at least $20 \%$ of all white dwarfs in a local volume of $20 \mathrm{pc}$ have magnetic fields, and that magnetism is not a common phenomenon for white dwarfs. The observations were carried out at FORS2/VLT and the work emphasizes the enduring importance of spectropolarimetry in the search for and study of new magnetic white dwarfs, especially old, cold, and faint. 


\subsection{Active Early-and Late-Type Stars with Complex Fields}

The paper by Petit et al. (2021) is devoted to the study of the magnetic field and activity of the dwarf $\mathrm{K} 2 \epsilon$ Eri using simultaneous observations with the SPIRou and NARVAL instruments, as well as with the TESS space telescope. The fundamental parameters of the star, determined from the spectropolarimetry data in the visible and near-IR ranges, coincide. It was found that the surface magnetic field of the star reaches $1.9 \mathrm{kG}$, the filling factor is $f=12.5 \pm 1.7 \%$. The polarimetric data, chromospheric emission, and broadband photometry display clear signs of nonrotational evolution over the course of data collection. The characteristic decay times, deduced from the light curve and the longitudinal magnetic field, are 3040 days. The evolution of the magnetic geometry occurs in $57 \pm 5$ days. The structure of the field is dominated by the toroidal component.

In Metcalfe et al. (2019), spectropolarimetry of old solar-type stars was performed on LBT/PEPSI. They are born with a rapid rotation and strong magnetic fields. In the course of evolution, due to magnetic braking, rotation slows down. The degree of the angular momentum loss depends on the morphology of the magnetic field. There is evidence of a significant decrease in magnetic braking in the middle of a star's life on the MS. To test this assumption, two stars were studied: the more active HD 100180 with a nonaxisymmetric magnetic field and the less active HD 143761. According to the authors, the astroseismic age of this star in the subsequent TESS observations will be significantly greater than the assumed gyrochronological age. It is concluded that there is a shift in the magnetic topology, which indicates the angular momentum loss in middle-aged stars.

Kochukhov et al. (2020) studied the Zeeman line broadening of 15 solar-like stars in order to detect a magnetic field. A total of 78 measurements were made. The authors found that the average field value lie within $1.3-2.0 \mathrm{kG}$ for the stars younger than $120 \mathrm{Myr}$; up to $0.2-0.8 \mathrm{kG}$-for older ones. The mean field strength clearly correlates with the Rossby number, coronal, and chromospheric activity determined from line emissions. The results show that for all stars the local field strength has the same value, about $3.2 \mathrm{kG}$ with a filling factor systematically increasing with stellar activity. The analysis was carried out by the Zeeman-Doppler imaging method.

Lavail et al. (2020) studied the large-scale magnetic field of the young binary system V1878 Ori. Both components are stars of the $\tau$ Tau type with an elongated and asynchronous orbit. Spectropolarimetric observations were made with ESPaDOnS. Using the LSD method, a circular polarization signal from both components was detected for many lines. Using the ZDI method, the authors constructed maps of the brightness distribution and magnetic field topology for both stars. It was found that components (A) and (B) of the system have completely different values and topology of the magnetic field: the magnetic field of the main component is predominantly poloidal and nonaxisymmetric with an average value of about $180 \mathrm{G}$, while the secondary component is predominantly toroidal and has an axisymmetric global field with an average value of $310 \mathrm{G}$.

Hubrig et al. (2020c) investigate the magnetic field of two Wolf-Rayet stars, WR 46 and WR 55, using spectropolarimetric observations at FORS2. WR 55 has a magnetic field of several hundred G. The star is located in the RCW 78 ring nebula and the molecular environment. No magnetic field was found for the secondary star.

Cole-Kodikara et al. (2019) studied the evolution of spots on the star LQ Hya which is a young analogue of the Sun. The object was observed with the SOFIN spectrograph for more than 25 years. The Doppler imaging technique was used to derive the surface temperature maps. A weak but systematic increase in the temperature of the star's surface was found. High-latitude sunspots are clearly asymmetric. Equatorial spots are also visible, but their interpretation is difficult due to poor coverage of observations in the phase of the star's rotation period. It is concluded that the sunspot activity is observed at high latitudes and the equator, while at temperate latitudes it is weaker.

Aronson and Piskunov (2019) introduced a modelfree method for mapping surface brightness variations. The method does not depend on stellar atmosphere models or limb-darkening equations. The method is based on the analysis of high-quality light curves from several transit events and light variations are interpreted as brightness variations on the stellar surface. No assumptions about the size, shape, or contrast of brightness variations are made.

Moura et al. (2020) carried out a spectroscopic analysis of two Herbig Ae/Be stars: HD 261941 and V590 Mon. Herbig stars are more massive than $\tau$ Tautype objects. The investigated objects have the detected magnetic fields. The authors found that there is enough circumstellar material in the vicinity of these two stars to make accretion or ejection possible. Using magnetohydrodynamic models, it is shown that in the case of HD 261941, outflow and accretion take place, and for V590 Mon, the $H_{\alpha}$ line origins mainly in the stellar wind from the disk.

Lisogorskyi et al. (2020) consider the effect of stellar activity on the accuracy of radial velocity measurements in exoplanet searches. Simulation is carried out under the assumption of a different set of unresolved magnetic spots in a rotating star of the K2 $\epsilon$ Eri type. The signal from the magnetic spots gives a value of the order of $10 \mathrm{~m} \mathrm{~s}^{-1}$ in the amplitude of the radial velocity variations. The shape of the rotation curve depends on the distribution of spots. The authors emphasize that understanding the stellar magnetic activity and spot 
distribution is crucial for the detection of Earth analogues.

Cang et al. (2020) studied the magnetic field of a young solar analog V530 Per. The object exhibits very strong magnetic activity showing the angular momentum loss thanks to the wind and mass ejections. V530 Per is the ultra-rapid rotator, a member of the young open cluster $\alpha$ Per. The rotation period is shorter than that of all other stars with available magnetic maps. Spectropolarimetric observations were performed with ESPaDOnS/CFHT over two nights. Using the Doppler-Zeeman imaging method, the authors reconstructed the surface brightness and large-scale magnetic field. The results of the work are as follows: the brightness map is dominated by a large, dark spot near the pole accompanied by a complex distribution of bright and dark features at lower latitudes. Most of the large-scale magnetic energy is concentrated in the toroidal component of the field. The main radial field structure is a positive region of about $+500 \mathrm{G}$ and is located in the region of the dark polar spot, the $H_{\alpha}$ line is observed in emission and is modulated with a rotation period.

Aurière et al. (2021) carried out the study of the red giant Pollux to characterize its magnetic field and infer how magnetic activity affects the radial velocity variations of a star, the period of which is about 590 days. With the use of ESPaDOnS/CFHT and NARVAL/TBL, they obtained spectropolarimetric series of the star within a range of 4.25 years, i.e., two periods of radial velocity variations. The LSD method was used to determine the magnetic field, and the ZDI method was used to construct the maps. As a result, it was found that the longitudinal magnetic field vary with a sinusoidal behavior with a period similar to the radial velocity variations. However, the period obtained from the ZDI maps is different: $P=660 \pm 15$ days. The poloidal component dominates and it is almost purely dipolar with an inclination of the dipole axis of 105 to the rotation axis. The average strength of the magnetic field at the pole is $0.44 \mathrm{G}$. The authors believe that the field of the star is formed by a dynamo mechanism. To explain the radial velocity variations, two scenarios are proposed: the presence of a planetary system and the magnetic activity.

In the paper by Das et al. (2019), coherent radio emission from the magnetic star HD 35298 was found. This is the fifth hot $\mathrm{Bp}$ star with the detected radio emission. HD 35298 is the slowest rotating and most distant of all Bp stars, in which radio emission has been detected.

Das et al. (2020) report that HD 133880 is the sixth hot magnetic star known to produce coherent pulsed radio emission by the process of electron cyclotron maser emission. Observations have shown that the properties of pulsations depend on the orientation of the star with respect to the line of sight. This result indicates a strong azimuthal asymmetry of the matter distribution in the magnetosphere relative to the magnetic field axis and a large inclination angle of the magnetic axis to the rotation axis, as well as the deviation of the stellar magnetic field from a dipolar topology.

It is shown by Krtička et al. (2020) that magnetic stars with strong helium lines can have surface distortions. They are caused by variations in the helium abundance over the surface and changes in the atmospheric height scale, which cause helium-rich regions to be dented inwards. Using $\alpha$ Cen as an example, the $\mathrm{He}, \mathrm{N}, \mathrm{O}, \mathrm{Si}$, and $\mathrm{Fe}$ distribution maps were derived using the Doppler mapping method. The authors found that the redistribution of the flux of a star with the non-uniform surface is due to the inhomogeneous distribution of helium and iron over its surface has the greatest effect on the UV and visible radiation.

Seach et al. (2020) presents the results of spectropolarimeric observations of 55 stars including $53 \mathrm{~F}$ type stars (from F0 to F9) plus two CP stars, $\beta$ CrB and $\delta$ Cap. High-resolution spectropolarimetry was carried out using ESPaDOnS/CFHT, NARVAL/TBL, and HARPSpol/3.6-m ESO. The authors look for magnetic fields in stars spanning a range of effective temperatures, where the transition from fossil to dynamo magnetic fields is believed to occur. A longitudinal magnetic field has been detected in 14 stars, in all spectral subclasses $\mathrm{F} 3 \mathrm{~V}-\mathrm{F} 9 \mathrm{~V}$. Its value ranges from $0.3 \pm 0.1 \mathrm{G}$ (36 UMa,F8V) to $8.3 \pm 0.9 \mathrm{G}(\chi \mathrm{Dra}$, $\mathrm{F} 8 \mathrm{~V})$. Thus, photospheric magnetic fields are found in stars as early as spectral type F3V with an outer convection zone thickness smaller than a few per cent of the stellar radius.

Klein et al. (2021) studied the large-scale magnetic field of the Proxima Centauri star near its maximum activity. Proxima Centauri is the closest star, a slowly rotating fully convective $\mathbf{M}$ dwarf. Ten circularly polarized spectra were obtained with HARPS from April to July 2017, which made it possible to determine the rotation period of the star: $P=89.8 \pm 4.0$ days. The ZDI method was used to derive the surface magnetic map. According to the results obtained, Proxima Centauri has a large-scale magnetic field with a typical strength of $200 \mathrm{G}$. Field topology is mostly poloidal, moderately axisymmetric. The dipole component of $135 \mathrm{G}$ is inclined at an angle of $51^{\circ}$ to the rotation axis. The large-scale magnetic flux is roughly 3 times smaller than the flux measured from the Zeeman broadening of the unpolarized lines. This leads to the assumption that the underlying dynamo is efficient at generating a magnetic field at the largest spatial scales.

\section{CONCLUSIONS}

We provide an overview of over 70 papers published in 2020. Of these, 26 are published in MNRAS, 11-in Astronomy and Astrophysics, 7-in Astrophysical Bulletin, the rest-in other leading journals and conference proceedings. We can conclude that MNRAS 
remains to be the world's leading journal, in which the results of studies of magnetic stars are published. Note that the number of studies on magnetic white dwarfs and classical chemically peculiar stars is lower than in previous years, but interest in studying the magnetic field of active solar-like stars has increased. Radio emission was found from several magnetic B stars. In 2020, 6-7 research groups continued active studies of stellar magnetism using the world's largest telescopes (8-m VLT, 6-m BTA, and $3.6 \mathrm{~m}$ CFHT), developed and introduced new methods of data analysis. Thus, studies of stellar magnetism continue to be relevant.

\section{ACKNOWLEDGMENTS}

The author thanks A.V. Moiseeva for help in preparing the manuscript.

\section{FUNDING}

The author is grateful to the Russian Science Foundation for the partial financial support of the work (project no. 21-12-00147).

\section{CONFLICT OF INTERESTS}

The author declares no conflicts of interest.

\section{REFERENCES}

1. S. Alexeeva, T. Chen, T. Ryabchikova, et al., Astrophys. J. 896 (1), 59 (2020).

2. E. Aronson and N. Piskunov, Astron. and Astrophys. 630, A122 (2019).

3. M. Aurière, P. Petit, P. Mathias, et al., Astron. and Astrophys. 646, A130 (2021).

4. S. Bagnulo and J. D. Landstreet, Astron. and Astrophys. 643, A134 (2020).

5. S. Bagnulo, G. A. Wade, Y. Nazé, et al., Astron. and Astrophys. 635, A163 (2020).

6. K. Bernhard, S. Hümmerich, and E. Paunzen, Monthly Notices Royal Astron. Soc. 493 (3), 3293 (2020).

7. A. Blazère, P. Petit, C. Neiner, et al., Monthly Notices Royal Astron. Soc. 492 (4), 5794 (2020).

8. D. M. Bowman and D. L. Holdsworth, Astron. and Astrophys. 629, A21 (2019).

9. T. E. Burlakova, G. G. Valyavin, V. N. Aitov, et al., Astrophysical Bulletin 75 (4), 482 (2020).

10. T. Q. Cang, P. Petit, J. F. Donati, et al., Astron. and Astrophys. 643, A39 (2020).

11. G. Catanzaro, M. Giarrusso, M. Munari, and F. Leone, Monthly Notices Royal Astron. Soc. 499 (3), 3720 (2020).

12. E. M. Cole-Kodikara, M. J. Käpylä, J. J. Lehtinen, et al., Astron. and Astrophys. 629, A120 (2019).

13. B. Das, P. Chandra, M. E. Shultz, and G. A. Wade, Monthly Notices Royal Astron. Soc. 489 (1), L102 (2019).
14. B. Das, P. Chandra, and G. A. Wade, Monthly Notices Royal Astron. Soc. 499 (1), 702 (2020).

15. A. David-Uraz, V. Petit, M. E. Shultz, et al., Monthly Notices Royal Astron. Soc. 501 (2), 2677 (2021).

16. I. Di Varano, S. Yuan, M. Woche, et al., in Society of Photo-Optical Instrumentation Engineers (SPIE) Conference Series, Society of Photo-Optical Instrumentation Engineers (SPIE) Conference Series, vol. 11451, p. $114514 \mathrm{G}(2020)$.

17. J. F. Donati, D. Kouach, C. Moutou, et al., Monthly Notices Royal Astron. Soc. 498 (4), 5684 (2020).

18. Y. V. Glagolevskij, Astrophysical Bulletin 75 (2), 139 (2020).

19. M. J. Hobson, F. Bouchy, N. J. Cook, et al., Astron. and Astrophys. 648, A48 (2021).

20. D. L. Holdsworth and E. Brunsden, Publ. Astron. Soc. Pacific 132 (1016), 105001 (2020).

21. S. Hubrig, S. P. Järvinen, H. Korhonen, et al., Monthly Notices Royal Astron. Soc. 495 (1), L97 (2020a).

22. S. Hubrig, S. P. Järvinen, M. Schöller, and C. A. Hummel, MonthlyNotices Royal Astron. Soc. 491 (1), 281 (2020b).

23. S. Hubrig, M. Schöller, A. Cikota, and S. P. Järvinen, Monthly Notices Royal Astron. Soc. 499 (1), L116 (2020c).

24. S. Hubrig, M. Schöller, and S. P. Järvinen, in IAU General Assembly, pp. 132-132 (2020d).

25. S. Hümmerich, E. Paunzen, and K. Bernhard, Astron. and Astrophys. 640, A40 (2020).

26. S. P. Järvinen, S. Hubrig, M. Schöller, et al., Monthly Notices Royal Astron. Soc. 501 (3), 4534 (2021).

27. Z. Keszthelyi, G. Meynet, M. E. Shultz, et al., Monthly Notices Royal Astron. Soc. 493 (1), 518 (2020).

28. A. F. Kholtygin, A. V. Moiseeva, I. A. Yakunin, and S. Hubrig, Astrophysical Bulletin 75 (3), 284 (2020).

29. B. Klein, J.-F. Donati, É. M. Hébrard, et al., Monthly Notices Royal Astron. Soc. 500 (2), 1844 (2021).

30. O. Kochukhov, T. Hackman, J. J. Lehtinen, and A. Wehrhahn, Astron. and Astrophys. 635, A142 (2020).

31. J. Krtička, Z. Mikulášek, M. Prvák, et al., Monthly Notices Royal Astron. Soc. 493 (2), 2140 (2020).

32. D. W. Kurtz, S. Hubrig, S. P. Järvinen, and M. Schöller, Research Notes of the American Astronomical Society 4 (9), 157 (2020).

33. J. D. Landstreet and S. Bagnulo, Astron. and Astrophys. 634, L10 (2020).

34. J. D. Landstreet, A. David-Uraz, O. Kochukhov, et al., in C. Neiner, W. W. Weiss, D. Baade, (eds.), Stars and their Variability Observed from Space, pp. 181-182 (2020).

35. A. Lavail, O. Kochukhov, G. A. J. Hussain, et al., Monthly Notices Royal Astron. Soc. 497 (1), 632 (2020).

36. M. Lisogorskyi, S. Boro Saikia, S. V. Jeffers, et al., Monthly Notices Royal Astron. Soc. 497 (3), 4009 (2020).

37. A. Marconi, M. Abreu, V. Adibekyan, et al., The Messenger 182, 27 (2021). 
38. G. Mathys, V. Khalack, and J. D. Landstreet, Astron. and Astrophys. 636, A6 (2020).

39. T. S. Metcalfe, O. Kochukhov, I. V. Ilyin, et al., Astrophys. J. 887 (2), L38 (2019).

40. Z. Mikulášek, J. Krtička, M. E. Shultz, et al., in G. Wade, E. Alecian, D. Bohlender, and A. Sigut (eds.), Stellar Magnetism: A Workshop in Honour of the Career and Contributions of John D. Landstreet, vol. 11, pp. 46-53 (2020a).

41. Z. Mikulášek, E. Paunzen, S. Hümmerich, et al., Monthly Notices Royal Astron. Soc. 498 (1), 548 (2020b).

42. T. Moura, S. H. P. Alencar, A. P. Sousa, et al., Monthly Notices Royal Astron. Soc. 494 (3), 3512 (2020).

43. K. E. Nielsen, K. G. Carpenter, G. V. Kober, and G. M. Wahlgren, Astrophys. J. 899 (2), 166 (2020).

44. E. Niemczura, P. A. Kołaczek-Szymań ski, F. Castelli, et al., in F. Salama and H. Linnartz (eds.), Laboratory Astrophysics: From Observations to Interpretation, vol. 350, pp. 412-414 (2020).

45. E. Paunzen, J. Janík, J. Krtička, et al., in C. Neiner, W. W. Weiss, D. Baade, (eds.), Stars and their Variability Observed from Space, pp. 19-24 (2020).

46. K. Perraut, M. Cunha, A. Romanovskaya, et al., Astron. and Astrophys. 642, A101 (2020).

47. P. Petit, C. P. Folsom, J. F. Donati, et al., Astron. and Astrophys. 648, A55 (2021).

48. V. Petit, G. A. Wade, F. R. N. Schneider, et al., Monthly Notices Royal Astron. Soc. 489 (4), 5669 (2019).

49. N. Piskunov, A. Wehrhahn, and T. Marquart, Astron. and Astrophys. 646, A32 (2021).

50. A. M. Romanovskaya, T. A. Ryabchikova, and D. V. Shulyak, Astronomy Letters 46 (5), 331 (2020).

51. A. M. Romanovskaya and D. V. Shulyak, INASAN Science Reports 3, 149 (2019).

52. I. I. Romanyuk, Astrophysical Bulletin 70 (2), 191 (2015).

53. I. I. Romanyuk, Astrophysical Bulletin 75 (4), 447 (2020).

54. I. I. Romanyuk, D. O. Kudryavtsev, A. V. Moiseeva, and I. A. Yakunin, in I. I. Romanyuk, I. A. Yakunin, A. F. Valeev, and D. O. Kudryavtsev (eds.), GroundBased Astronomy in Russia. 21st Century, pp. 301-303 (2020a).

55. I. I. Romanyuk, D. O. Kudryavtsev, E. A. Semenko, et al., in I. I. Romanyuk, I. A. Yakunin, A. F. Valeev, and D. O. Kudryavtsev (eds.), Ground-Based Astronomy in Russia. 21st Century, pp. 56-63 (2020b).

56. I. I. Romanyuk, Z. Mikulášek, S. Hümmerich, et al., in C. Neiner, W. W. Weiss, D. Baade, (eds.), Stars and their Variability Observed from Space, pp. 197-198 (2020c).

57. I. I. Romanyuk, A. V. Moiseeva, E. A. Semenko, et al., Astrophysical Bulletin 75 (3), 294 (2020d).

58. I. I. Romanyuk, E. A. Semenko, A. V. Moiseeva, et al., Astrophysical Bulletin 76 (1), 39 (2021).

59. I. I. Romanyuk, I. A. Yakunin, A. F. Valeev, and D. O. Kudryavtsev (eds.), Ground-Based Astronomy in Russia. 21st Century (2020e).

60. I. S. Savanov, I. I. Romanyuk, and E. S. Dmitrienko, Astronomicheskij Tsirkulyar 1647, 1 (2020).

61. M. Schöller, C. A. Hummel, S. Hubrig, et al., Astron. and Astrophys. 642, A188 (2020).

62. J. M. Seach, S. C. Marsden, B. D. Carter, et al., Monthly Notices Royal Astron. Soc. 494 (4), 5682 (2020).

63. E. A. Semenko, I. I. Romanyuk, I. A. Yakunin, et al., in G. Wade, E. Alecian, D. Bohlender, and A. Sigut (eds.), Stellar Magnetism: A Workshop in Honour of the Career and Contributions of John D. Landstreet, vol. 11, pp. 25-31 (2020).

64. M. E. Shultz, A. David-Uraz, J. Labadie-Bartz, et al., in C. Neiner, W. W. Weiss, D. Baade, (eds.), Stars and their Variability Observed from Space, pp. 177-179 (2020a).

65. M. E. Shultz, C. Johnston, J. Labadie-Bartz, et al., Monthly Notices Royal Astron. Soc. 490 (3), 4154 (2019a).

66. M. E. Shultz, S. Owocki, T. Rivinius, et al., Monthly Notices Royal Astron. Soc. 499 (4), 5379 (2020b).

67. M. E. Shultz, G. A. Wade, T. Rivinius, et al., Monthly Notices Royal Astron. Soc. 490 (1), 274 (2019b).

68. M. Skarka, P. Kabáth, E. Paunzen, et al., in C. Neiner, W. W. Weiss, D. Baade, (eds.), Stars and their Variability Observed from Space, pp. 175-176 (2020).

69. G. G. Valyavin, F. A. Musaev, A. V. Perkov, et al., Astrophysical Bulletin 75 (2), 191 (2020a).

70. G. G. Valyavin, A. V. Perkov, F. A. Musaev, et al., in I. I. Romanyuk, I. A. Yakunin, A. F. Valeev, and D. O. Kudryavtsev (eds.), Ground-Based Astronomy in Russia. 21st Century, pp. 41-47 (2020b).

71. G. A. Wade, S. Bagnulo, Z. Keszthelyi, et al., Monthly Notices Royal Astron. Soc. 492 (1), L1 (2020).

72. M. Weber, M. Woche, K. G. Strassmeier, et al., in Society of Photo-Optical Instrumentation Engineers (SPIE) Conference Series, Society of Photo-Optical Instrumentation Engineers (SPIE) Conference Series, vol. 11449, p. $114490 \mathrm{~J}$ (2020).

Translated by N. Oborina 